\title{
Óleo de cravo como anestésico em adultos de tilápia-do-nilo
}

\author{
Larissa Novaes Simões ${ }^{(1)}$, Genilson Paiva(1) e Levy de Carvalho Gomes ${ }^{(1)}$
}

${ }^{(1)}$ Centro Universitário Vila Velha, Laboratório de Ecotoxicologia Aquática, Rua Comissário José Dantas de Melo, oㅡ 21, CEP 29102-770

Vila Velha, ES. E-mail: larissanovaess@hotmail.com, genilson.paiva@uvv.br, levy.gomes@uvv.br

Resumo - O objetivo deste trabalho foi testar a eficiência do óleo de cravo como anestésico em adultos de tilápia-do-nilo (Oreochromis niloticus) e avaliar, sensorialmente, o aroma e o sabor do filé, após a anestesia. No primeiro experimento, os peixes foram expostos a banhos anestésicos em diferentes concentrações de óleo de cravo $\left(0,100,150,200,250\right.$ e $\left.350 \mathrm{mg} \mathrm{L}^{-1}\right)$. No segundo experimento, avaliaram-se diferentes tempos $(10,20$ e 30 minutos) de exposição à anestesia. Finalmente, o aroma e o sabor dos filés de tilápia foram testados, em diferentes tempos de exposição à concentração adequada de óleo de cravo. A concentração de $250 \mathrm{mg} \mathrm{L}^{-1}$ de óleo de cravo foi adequada para a indução de parada dos batimentos operculares em adultos de tilápia, e para a anestesia voltada para biometria e breve manejo, a concentração recomendada é $100 \mathrm{mg} \mathrm{L}^{-1}$. Os filés de tilápias previamente anestesiadas com óleo de cravo apresentaram diferença moderada no aroma e no sabor logo após a anestesia. O óleo de cravo é um anestésico eficaz no manejo de adultos de tilápia em procedimentos de rotina na piscicultura, porém o abate de tilápias deve ser realizado a partir de 12 horas após a exposição a este anestésico, para não induzir alteração nas características organolépticas dos filés de peixes anestesiados.

Termos para indexação: Oreochromis niloticus, análise sensorial, filés, sedação.

\section{Clove oil as an anesthetic on Nile tilapia adults}

\begin{abstract}
The objective of this study was to test the efficiency of clove oil as an anesthetic for adult tilapia (Oreochromis niloticus) and to evaluate, sensorially, the aroma and taste of fillets after anesthesia. In the first experiment, fish were exposed to different clove oil concentrations $\left(0,100,150,200,250\right.$ and $\left.350 \mathrm{mg} \mathrm{L}^{-1}\right)$ of anesthetic baths. In the second experiment, different times of exposure (10, 20 and 30 minutes) to anesthesia were evaluated. Finally, the aroma and taste of the tilapia fillets, when exposed to optimal concentration of clove oil, were tested. The concentration of $250 \mathrm{mg} \mathrm{L}^{-1}$ of clove oil was adequate to induce lower opercular movement in adult tilapia, and for anesthesia for biometrics and brief management, the recommended concentration is $100 \mathrm{mg} \mathrm{L}^{-1}$. Fillets from tilapia anesthetized with clove oil showed a moderate difference in aroma and taste just after anesthesia. Thus, clove oil is an effective anesthetic for the management of adult tilapia as a routine procedure in fish farming, however tilapias should be slaughtered 12 hours after exposure to the drug, so as not to change the organoleptic characteristics of anesthetized fishes.
\end{abstract}

Index terms: Oreochromis niloticus, sensory analysis, fillets, sedation.

\section{Introdução}

A criação de tilápias-do-nilo (Oreochromis niloticus L., 1758) tem crescido, e esta é, atualmente, a terceira espécie de maior importância econômica na aquicultura mundial (Watanabe et al., 2002). O Brasil ocupa o sexto lugar na produção de tilápia, e é responsável por 3,3\% do total da produção (Food and Agriculture Organization of the United Nations, 2007). A tilapicultura é reconhecida como uma importante atividade agroindustrial, capaz de gerar grande retorno financeiro para os produtores (Pinheiro et al., 2006).

$\mathrm{O}$ aumento na produção da tilápia se deve às características da carne, ao elevado valor nutricional, às excelentes características organolépticas e à ausência de espinhos intermusculares em forma de Y. Do ponto de vista da criação, é um peixe de rápido desenvolvimento, facilmente reproduzido em cativeiro, e que atinge tamanho comercial em poucos meses de criação, o que pode proporcionar mais de um ciclo de produção por ano (Souza \& Maranhão, 2001).

Um dos procedimentos mais importantes em pisciculturas é a anestesia, utilizada para facilitar o manejo, evitar a possibilidade de ferimentos e, possivelmente, reduzir o estresse dos peixes (Small, 2004). A anestesia de adultos de tilápia durante o manuseio é importante, já que os raios duros que formam a nadadeira dorsal facilmente ocasionam 
ferimentos aos operadores e aos outros peixes, caso não estejam anestesiados (Simões \& Gomes, 2009).

De acordo com Bosworth et al. (2007), o peixe sedado dentro do processo de captura, antes do abate, evita o estresse que supostamente ocorreria durante o manejo e abate, consequentemente, melhorando a qualidade da carne. Entretanto, existe preocupação de que resíduos anestésicos possivelmente deixados na carne possam alterar as características organolépticas - aroma e sabor - naturais do peixe, a ponto de resultar na rejeição do produto pelos consumidores (Olsen et al., 2006; Ribas et al., 2007).

O óleo de cravo é um produto natural resultante da destilação das folhas, caule, flores e brotos das árvores Syzygium aromaticum (L.) Merr. \& L.M. Perry e Myrcianthes fragrans (Sw.) McVaugh, cujo constituinte majoritário é o eugenol, que representa de 70 a $90 \%$ do óleo (Mazzafera, 2003). Estudos têm demonstrado a eficiência do óleo de cravo e do eugenol em anestesiar peixes de clima tropical, como o tambaqui (Roubach et al., 2005) e o matrinxã (Vidal et al., 2008), e de clima temperado, como a truta (Velísek et al., 2005) e o salmão (Iversen et al., 2003).

Além disso, estudos conduzidos com perca-prateada [Bidyanus bidyanus (Mitchell, 1838)] demonstraram que o tempo residual desses produtos no filé do peixe é inferior a 24 horas (Kildea et al., 2004). Essas características fazem com que o isoeugenol, na forma de AQUI-S, seja liberado para anestesia de peixes em alguns países como Austrália, Nova Zelândia e Chile (Bosworth et al., 2007; Ross \& Ross, 2008).

O objetivo deste trabalho foi testar a eficiência do óleo de cravo como anestésico em adultos de tilápia-do-nilo (Oreochromis niloticus), durante o manejo e avaliar, sensorialmente, os parâmetros organolépticos, aroma e sabor, do filé, após a anestesia.

\section{Material e Métodos}

Para a determinação da concentração de anestésico adequada, adultos de tilápia $(\mathrm{n}=70 ; 670,00 \pm 75,28 \mathrm{~g}$ e 28,32 $\pm 0,92 \mathrm{~cm}$ ) foram obtidos na Fazenda Barra do Mangaraí, Santa Leopoldina, ES, e transferidos para a Associação dos Aquicultores do Espírito Santo, Cariacica, ES, onde foram colocados para aclimatação em viveiros de $20 \mathrm{~m}^{2}$, com troca de água constante. Nesse local e sob essas condições, os peixes permaneceram por aproximadamente dez dias.

Durante a aclimatação, a cada três dias, a qualidade da água foi monitorada. Nesse período, os peixes foram alimentados diariamente com ração comercial para peixes contendo $22 \%$ de proteína bruta, Propeixe $22 \%$ E (Nutriave Alimentos Ltda., Viana, ES, Brasil). Os ensaios foram realizados em aquários de plástico de $45 \mathrm{~L}$, contendo $20 \mathrm{~L}$ de água e a recuperação foi sempre realizada em aquários de plástico de $45 \mathrm{~L}$, contendo 25 $\mathrm{L}$ de água, com aeração constante. A água do aquário foi trocada ao término de cada ensaio.

Antes de ser utilizado, o óleo de cravo (Petite Marie, Itaquaquecetuba, SP, Brasil), na densidade de $1,03 \mathrm{~g} \mathrm{~cm}^{-3}$, foi diluído para uma concentração de $1 \mathrm{~mL}$ por $10 \mathrm{~mL}$ em etanol a $95 \%$ para o preparo da solução-mãe. Foram testadas cinco concentrações de óleo de cravo - 100, 150, 200, 250 e $300 \mathrm{mg} \mathrm{L}^{-1}$. Oito peixes foram expostos a cada uma das concentrações do anestésico, de forma individual, para se observar a recuperação e o tempo de indução a cada estágio de anestesia, reproduzido por eventos comportamentais (Tabela 1). Após dez minutos de exposição ao óleo de cravo, cada peixe foi removido para o aquário de recuperação. Os estágios avaliados de indução à anestesia seguiram os critérios propostos por Stoskopf (1993): perda de reação a estímulos, perda parcial

Tabela 1. Tempo, em segundos, de indução aos estágios de anestesia de adultos de tilápia-do-nilo (Oreochromis niloticus) expostos a diferentes concentrações de óleo de cravo $^{(1)}$.

\begin{tabular}{|c|c|c|c|c|c|c|}
\hline \multirow{2}{*}{$\begin{array}{l}\text { Óleo de cravo } \\
\left(\mathrm{mg} \mathrm{L}^{-1}\right)\end{array}$} & \multicolumn{6}{|c|}{ Evento comportamental (segundos) } \\
\hline & $\begin{array}{c}\text { Perda de reação à } \\
\text { estímulos }\end{array}$ & $\begin{array}{l}\text { Perda parcial de } \\
\text { equilíbrio }\end{array}$ & $\begin{array}{l}\text { Perda total } \\
\text { de equilíbrio }\end{array}$ & $\begin{array}{c}\text { Redução dos } \\
\text { batimentos operculares }\end{array}$ & $\begin{array}{c}\text { Parada dos } \\
\text { batimentos operculares }\end{array}$ & Recuperação \\
\hline 100 & $13,1 \pm 0,6 \mathrm{a}$ & $23,9 \pm 1,0 \mathrm{a}$ & $92,1 \pm 4,4 \mathrm{ab}$ & $151,3 \pm 7,2 \mathrm{a}$ & $-{ }^{(2)}$ & $503,9 \pm 35,5 \mathrm{a}$ \\
\hline 150 & $14,3 \pm 1,0 \mathrm{a}$ & $27,6 \pm 1,2 b$ & $68,4 \pm 2,9 b$ & $100,8 \pm 1,7 \mathrm{bc}$ & $524,0 \pm 18,3 \mathrm{a}$ & $587,4 \pm 34,1 \mathrm{ab}$ \\
\hline 200 & $10,1 \pm 0,4 \mathrm{a}$ & $16,8 \pm 0,7 \mathrm{~cd}$ & $97,4 \pm 9,6 \mathrm{a}$ & $127,1 \pm 8,7 \mathrm{ac}$ & $229,5 \pm 11,8 b$ & $580,6 \pm 42,2 \mathrm{ab}$ \\
\hline 250 & $8,9 \pm 0,4 \mathrm{~b}$ & $14,1 \pm 0,6 \mathrm{c}$ & $71,4 \pm 8,7 \mathrm{ab}$ & $94,4 \pm 9,9 \mathrm{~b}$ & $192,5 \pm 15,6 \mathrm{~b}$ & $653,5 \pm 47,5 \mathrm{ab}$ \\
\hline 300 & $10,4 \pm 0,8 \mathrm{~b}$ & $18,5 \pm 0,9 \mathrm{~d}$ & $67,1 \pm 6,5 b$ & $81,8 \pm 6,7 \mathrm{~b}$ & $134,8 \pm 8,7 \mathrm{c}$ & $1.063,3 \pm 247,9 \mathrm{~b}$ \\
\hline
\end{tabular}

${ }^{(1)}$ Médias \pm erro-padrão seguidas por letras iguais, nas colunas, não diferem entre si pelo teste de Tukey, a $5 \%$ de probabilidade ${ }^{(2)}$ Os peixes não alcançaram este estágio de anestesia. 
de equilíbrio, perda total de equilíbrio, redução dos batimentos operculares e parada dos batimentos operculares. Foi considerado recuperado o peixe que retomava totalmente os movimentos e nadava ativamente no aquário após o tempo de efeito da anestesia.

Foi realizado um ensaio para verificar a margem de segurança do anestésico. Para isso foi considerado o resultado do primeiro experimento. Além disso, a concentração de $250 \mathrm{mg} \mathrm{L}^{-1}$ foi escolhida como a mais adequada para atingir todos os estágios de anestesia, pois o tempo para atingir a redução dos batimentos operculares é menor que nas demais concentrações, com exceção da concentração de $300 \mathrm{mg} \mathrm{L}^{-1}$.

Analisou-se o tempo de recuperação após indução à concentração de óleo de cravo por 10,20 e 30 minutos ( $\mathrm{n}=8$ para cada tempo de exposição) em aquários de plástico de $45 \mathrm{~L}$, contendo $20 \mathrm{~L}$ de água. Após a exposição, os peixes foram transferidos para o aquário de recuperação de $45 \mathrm{~L}$, contendo $25 \mathrm{~L}$ de água, com aeração constante. Foram avaliadas a recuperação e a mortalidade.

$\mathrm{Na}$ análise sensorial do filé de peixes anestesiados, 42 animais com $515,47 \pm 135,21 \mathrm{~g}$ e $28,97 \pm 3,44 \mathrm{~cm}$, foram colocados para aclimatação em um tanque de $4 \mathrm{~m}^{2}$, com sistema de aeração constante, por aproximadamente dez dias, na Agro Estância Lombardi, Ibiraçu, ES. A água do tanque foi mantida estática e foi inteiramente trocada a cada dois dias, sempre sifonando o fundo da caixa. Durante a aclimatação, a qualidade da água foi monitorada a cada dois dias. Neste período, os peixes foram alimentados diariamente com ração comercial para peixes contendo $22 \%$ de proteína bruta Propeixe $22 \%$ E (Nutriave Alimentos Ltda., Viana, ES, Brasil).

Os peixes foram mantidos sem alimentação 24 horas antes do experimento. Antes da anestesia, foram amostrados seis peixes como controle, não anestesiados. Após amostragem dos peixes controle, 36 peixes foram anestesiados, com $250 \mathrm{mg} \mathrm{L}^{-1}$ de óleo de cravo por dez minutos em aquários de plástico de $45 \mathrm{~L}$, contendo $20 \mathrm{~L}$ de água. Após a anestesia, os peixes foram transferidos para um tanque de $4 \mathrm{~m}^{2}$ com água corrente (vazão $=1 \mathrm{~L} \mathrm{~min}^{-1}$ ), e abatidos nos tempos 0 , ou logo após a anestesia, 12, 24, 48, 96 e 168 horas após a anestesia. Em cada tempo, foram analisados seis peixes.

Após a captura, as tilápias foram abatidas por hipotermia em caixas isotérmicas com gelo e água na razão $1: 1$, filetadas e embaladas individualmente em sacos de plástico transparentes e acondicionadas em uma caixa isotérmica contendo gelo. Após esses procedimentos, as tilápias foram transportadas para o Complexo Biopráticas do Centro Universitário de Vila Velha, ES, onde as amostras foram refrigeradas a $-20^{\circ} \mathrm{C}$, até o momento da análise sensorial.

Inicialmente, foram selecionadas 20 pessoas, que foram treinadas para reconhecimento dos diferentes aromas e sabores a serem avaliados. No treinamento foi utilizado um peixe de cada tratamento. $\mathrm{Na}$ análise sensorial, foi utilizado o Método de Comparação Múltipla, conforme Ferreira (2002).

Os filés, depois de descongelados, foram cortados em pedaços de aproximadamente $3 \mathrm{~cm}^{2}$ (10 g), embrulhados em folha de papel alumínio, submetidos ao forno convencional a $180^{\circ} \mathrm{C}$, por cinco minutos, sem tempero algum. $\mathrm{Na}$ análise do aroma, o embrulho era aberto e cheirado, e, em seguida, degustado, para avaliação do sabor. As amostras foram apresentadas simultaneamente, em bandejas de fundo claro e codificadas, sob condições laboratoriais e iluminação natural, a avaliadores que não interagiam entre si.

Os avaliadores foram solicitados a provar as amostras codificadas e identificar a intensidade da diferença do aroma e sabor - 0, nenhuma; 1, pequena; 2 , moderada e 3 , grande - em relação ao controle. Cada provador recebeu quatro amostras $(\mathrm{n}=5$ para cada tratamento $)$ de filé, sendo um controle e as outras três com anestésico.

Os parâmetros físico-químicos da água analisados apresentaram valores muito similares durante $o$ período de aclimatação e os ensaios estão descritos como uma média geral: oxigênio dissolvido: $6,53 \pm 0,92$ mg L ${ }^{-1}$; temperatura: $26,98 \pm 1,41^{\circ} \mathrm{C}$; condutividade: $63,34 \pm 2,87 \mu \mathrm{S} \mathrm{cm}^{-1}$; dureza: $20,69 \pm 6,43 \mathrm{mg} \mathrm{CaCo}_{3} \mathrm{~L}^{-1}$; pH: 7,00 $\pm 0,32$; e amônia: $0,04 \pm 0,01 \mathrm{mg} \mathrm{L}^{-1}$.

Nas análises estatísticas, os dados foram expressos em média e erro padrão. Os tempos para atingir os diferentes estágios de anestesia para as diferentes concentrações de óleo de cravo, assim como o tempo de recuperação após a exposição a diferentes tempos de anestesia, e a análise sensorial (aroma e sabor), foram avaliados por análise de variância (ANOVA), seguida do teste de Tukey, a 5\% de probabilidade. No experimento de análise sensorial, foram seguidos os critérios conforme Ferreira (2002). 


\section{Resultados e Discussão}

Com exceção da concentração de $100 \mathrm{mg} \mathrm{L}^{-1}$ de óleo de cravo, todas as demais concentrações avaliadas induziram os peixes a todos os estágios de anestesia. Entretanto, os peixes anestesiados com $250 \mathrm{mg} \mathrm{L}^{-1}$ alcançaram o estágio de redução dos batimentos operculares em um tempo significativamente menor que nas demais concentrações. A exceção foi a concentração de $300 \mathrm{mg} \mathrm{L}^{-1}$. Dessa forma, a concentração de $250 \mathrm{mg} \mathrm{L}^{-1}$ foi considerada adequada para indução de anestesia cirúrgica em adultos de tilápia.

Segundo Vidal et al. (2008), $75 \mathrm{mg} \mathrm{L}^{-1}$ de eugenol é a concentração adequada para induzir a perda total de movimento em juvenis de tilápia-do-nilo com peso aproximadamente de $5 \mathrm{~g}$. De acordo com Ross \& Ross (2008), a necessidade de maior concentração de anestésico para indução da anestesia em adultos de tilápia, como observado neste trabalho, tem relação com o tamanho: peixes menores normalmente necessitam de uma concentração menor de anestésico do que os maiores, padrão também observado por Woody et al. (2002) com o salmão-vermelho [Oncorhynchus nerka (Walbaum, 1792)], anestesiado com óleo de cravo. Outra provável diferença é o produto utilizado, uma vez que Vidal et al. (2008) utilizaram eugenol purificado.

A concentração adequada com finalidade de um breve manejo não necessita induzir a todos os estágios de anestesia, sendo necessário alcançar apenas a perda total de equilíbrio. A concentração de $100 \mathrm{mg} \mathrm{L}^{-1}$ foi escolhida por induzir os peixes à perda total de equilíbrio ao mesmo tempo que as demais concentrações, de modo a possibilitar o efeito desejado com a menor concentração possível (Tabela 1).

Em nenhuma das concentrações avaliadas foi observada mortalidade dos peixes, de maneira semelhante ao relatado por Inoue et al. (2003), que não observaram mortalidade de juvenis de matrinxã [Brycon cephalus (Gunther, 1869)], submetidos a até $70 \mathrm{mg} \mathrm{L}^{-1}$ de óleo de cravo. Esses resultados diferem dos que foram obtidos por Woody et al. (2002) com adultos de salmão [Oncorhynchus nerka (Walbaum, 1792)], que não recuperaram equilíbrio e morreram com concentrações de $110 \mathrm{mg} \mathrm{L}^{-1}$ de óleo de cravo-da-índia. A maior resistência de adultos de peixes tropicais ao anestésico pode estar relacionada à maior capacidade desses peixes em metabolizar os componentes do óleo de cravo, proporcionado pela maior temperatura corporal. De acordo com Baldisserotto (2009), a temperatura corporal afeta a fisiologia e a velocidade das reações químicas, havendo maior taxa metabólica em peixes de clima tropical, como a tilápia, e menor taxa metabólica em peixes de clima temperado, como o salmão.

O tempo de recuperação após a exposição por 10 min $(616,1 \pm 26,1 \mathrm{~s})$, a $250 \mathrm{mg} \mathrm{L}^{-1}$ de óleo de cravo foi significativamente menor do que a recuperação dos peixes expostos ao anestésico por $30 \mathrm{~min}$ $(2.070,0 \pm 229,9 \mathrm{~s})$. Os peixes anestesiados por $20 \min (918,4 \pm 32,6 \mathrm{~s})$ não apresentaram diferença significativa dos demais tempos de anestesia. Não foi observada mortalidade dos peixes expostos ao anestésico, em nenhum dos tempos avaliados, o que corrobora o estudo de Roubach et al. (2005), em que adultos de tambaqui [Colossoma macropomum (Cuvier, 1818)], expostos a concentrações de até $100 \mathrm{mg} \mathrm{L}^{-1} \mathrm{de}$ eugenol, não apresentaram mortalidade em contagens até 30 min de exposição.

Ross \& Ross (2008) relataram que 10 min deve ser o tempo máximo de anestesia em peixes. Entretanto, estudos com adultos de algumas espécies de peixes tropicais como o tambaqui (Roubach et al., 2005) e a tilápia demonstram que o óleo de cravo é seguro se usado em um tempo de até $30 \mathrm{~min}$ de anestesia. Esse tempo de anestesia, porém, deve ser evitado, pois a recuperação dos peixes é muito lenta, e chega a demorar três vezes mais do que em peixes anestesiados por $10 \mathrm{~min}$.

$\mathrm{Na}$ análise de variância do ensaio sensorial dos filés, o F calculado - razão de variância - foi maior que o $\mathrm{F}$ tabelado aos níveis de 5 e $1 \%$ de probabilidade, o que, conforme Ferreira (2002), determina que há diferença significativa entre as amostras tanto no aroma quanto no sabor (Tabela 2). As características organolépticas, aroma e sabor, de filés de tilápias expostas a $250 \mathrm{mg} \mathrm{L}^{-1}$ de óleo de cravo foram significativamente diferentes apenas logo após a anestesia (tempo de zero hora), sendo considerada moderadamente diferente pelos avaliadores. A partir de 12 horas após a anestesia, não se observou mais diferença significativa com relação a nenhuma das características avaliadas, o que indica que a partir deste tempo não há nenhuma diferença no aroma e no sabor dos filés, em relação a peixes não anestesiados. Cunha et al. (2010) também observaram aroma e sabor do eugenol em filé-de-jundiá [Rhamdia quelen (Qouy \& Gaimard, 1824)], abatido logo após a anestesia com $50 \mathrm{mg} \mathrm{L}^{-1}$ de eugenol. Porém, 
Kildea et al. (2004) relataram a eliminação do óleo de cravo do tecido de perca-prateada, 12 horas após a exposição a $50 \mathrm{mg} \mathrm{L}^{-1}$ desse anestésico. Os resultados obtidos no presente trabalho corroboram os dos autores já citados.

Segundo Gomes et al. (2001), não há legislação no Brasil sobre a utilização de anestésicos em peixes para consumo humano. Porém, países como os Estados Unidos já possuem regulamentação para o uso do MS-222 e benzocaína e a Nova Zelândia para o uso do Aqui-S e MS-222. Nos EUA, os animais anestesiados com MS-222 e benzocaína devem ser depurados por 21 dias; na Nova Zelândia, para o MS-222, são necessários dez dias de depuração e não existe período específico para a benzocaína. Os peixes anestesiados com Aqui-S, cujo principio ativo é o isoeugenol, não necessitam de depuração (Ross \& Ross, 2008). Os resultados obtidos neste e em outros estudos com espécies criadas no Brasil, como o matrinxã, lambari e a própria tilápia (Inoue et al., 2003; Oliveira et al., 2009; Pereira-da-Silva et al., 2009) indicam que o óleo de cravo e seus derivados são candidatos à liberação para uso em peixes; entretanto, deve haver rigor em relação ao tempo de abate após exposição ao anestésico.

Apesar de ser amplamente conhecido que o tempo residual do anestésico no tecido animal é baixo, o resultado obtido neste trabalho mostra que a anestesia com óleo de cravo altera a palatabilidade dos filés logo após a anestesia. De acordo com Santos et al. (2007), a palatabilidade do filé também é de extrema importância para que os consumidores fiquem satisfeitos com o produto. Ribas et al. (2007) relatam que o óleo

Tabela 2. Análise de variância para o aroma e o sabor de filés de tilápia-do-nilo (Oreochromis niloticus) após a anestesia com $250 \mathrm{mg} \mathrm{L}^{-1}$ de óleo de cravo ${ }^{(1)}$.

\begin{tabular}{lcccc}
\hline Fonte de variação & GL & SQ & QM & Fcal \\
\hline Avaliador & 9 & 4,15 & 0,46 & \\
Amostra & 5 & 14,28 & 2,86 & $6,93 * *$ \\
Resíduo & 45 & 18,55 & 0,41 & \\
\hline Total & 59 & 36,98 & & \\
\hline & 5 & \multicolumn{3}{c}{ Sabor } \\
Avaliador & 9 & 1,44 & 0,16 & \\
Amostra & 5 & 14,80 & 2,96 & \\
Resíduo & 45 & 15,36 & 0,34 & \\
\hline Total & 59 & 31,60 & & \\
\hline
\end{tabular}

${ }^{(1)} \mathrm{GL}$, grau de liberdade; SQ, soma dos quadrados; QM, quadrados médios; Fcal, razão de variância calculado. **Significativo a $1 \%$ de probabilidade. de cravo é o melhor método de atordoamento para linguado-senegalês [Solea senegalensis (Kaup, 1858)], porque não só garante boa qualidade final do produto, mas também é aceitável para o consumo humano direto. Dessa forma, recomenda-se, com base em critérios sensoriais, que adultos de tilápia destinados a filetagem, que foram anestesiados com a concentração adequada (250 $\mathrm{mg} \mathrm{L}^{-1}$ ), só sejam abatidos 12 horas após a exposição ao óleo de cravo.

\section{Conclusões}

1. O óleo de cravo é um anestésico eficaz para o manejo de adultos de tilápia em procedimentos de rotina na piscicultura.

2. As concentrações de $250 \mathrm{mg} \mathrm{L}^{-1}$ e $100 \mathrm{mg} \mathrm{L}^{-1}$ de óleo de cravo são adequadas para a indução de anestesia cirúrgica e de anestesia voltada para biometria e breve manejo, respectivamente.

3. A partir de 12 horas após a exposição de tilápias ao óleo de cravo, os peixes não apresentam aroma ou sabor diferenciado e, portanto, o abate pode ser realizado.

\section{Agradecimentos}

Ao Centro Universitário Vila Velha e à Fundação Nacional de Desenvolvimento do Ensino Superior Particular, pelo financiamento do trabalho; ao Conselho Nacional de Desenvolvimento Científico e Tecnológico, pela bolsa de pesquisa.

\section{Referências}

BALDISSEROTTO, B. Fisiologia de peixes aplicada à piscicultura. 2.ed. Santa Maria: UFSM, 2009. 352p.

BOSWORTH, B.G.; SMALL, B.C.; GREGORY, D.; KIM, J.; BLACK, S.; JERRETT, A. Effects of rested-harvest using the anesthetic AQUI-S ${ }^{\mathrm{TM}}$ on channel catfish, Ictalurus punctatus, physiology and fillet quality. Aquaculture, v.262, p.302-318, 2007.

CUNHA, M.A. da; ZEPPENFELD, C.C.; GARCIA, L. de O.; LORO, V.L.; FONSECA, M.B. da; EMANUELLI, T.; VEECK, A.P. de L.; COPATTI, C.E.; BALDISSEROTTO, B. Anesthesia of silver catfish with eugenol: time of induction, cortisol response and sensory analysis of fillet. Ciência Rural, v.40, p.2107-2114, 2010.

FERREIRA, S.M.R. Controle de qualidade em sistemas de alimentação coletiva I. São Paulo: Varela, 2002. 173p.

FOOD AND AGRICULTURE ORGANIZATION OF THE UNITED NATIONS. FishStat Plus: universal software for fishery statistical time series. Version 2.32. Rome: FAO, 2007. 
GOMES, L.C.; CHIPPARI-GOMES, A.R.; LOPES, N.P.; ROUBACH, R.; ARAÚJO-LIMA, C.A.R.M. Efficacy of benzocaine as an anesthetic in juvenile tambaqui, Colossoma macropomum. Journal of the World Aquaculture Society, v.32, p.426-431, 2001.

INOUE, L.KA.; SANTOS NETO, C.; MORAES, G. Clove oil as anaesthetic for juveniles of matrinxã Brycon cephalus (Gunther, 1869). Ciência Rural, v.33, p.943-947, 2003.

IVERSEN, M.; FINSTAD, B.; MCKINLEY, R.S.; ELIASSEN, R.A. The efficacy of metomidate, clove oil, Aqui-S ${ }^{\mathrm{TM}}$ and Benzoak ${ }^{\circledR}$ as anaesthetics in Atlantic salmon (Salmo salar L.) smolts, and their potential stress-reducing capackity. Aquaculture, v.221, p.549-566, 2003.

KILDEA, M.A.; ALLAN, G.L.; KEARNEY, R.E. Accumulation and clearance of the anaesthetics clove oil and AQUI-S ${ }^{\text {TM }}$ from the edible tissue of silver perch (Bidyanus bidyanus). Aquaculture, v.232, p.265-277, 2004.

MAZZAFERA, P. Efeito alelopático do extrato alcoólico do cravo-da-índia e eugenol. Revista Brasileira Botânica, v.26, p.231-238, 2003.

OLIVEIRA, J.R.; CARMO, J.L.; OLIVEIRA, K.K.C.; SOARES, M.C.F. Cloreto de sódio, benzocaína e óleo de cravo-da-índia na água de transporte de tilápia-do-nilo. Revista Brasileira de Zootecnia, v.38, p.1163-1169, 2009.

OLSEN, D.H.; SORENSENA, N.K.; STORMOA, S.K.; ELVEVOLLA, E.O. Effect of slaughter methods on blood spotting and residual blood in fillets of Atlantic salmon (Salmo salar). Aquaculture, v.258, p.462-469, 2006.

PEREIRA-DA-SILVA, E.M.; OLIVEIRA, R.H.F. de; RIBEIRO, M.A.R.; COPPOLA, M.P.. Efeito anestésico do óleo de cravo em alevinos de lambari. Ciência Rural, v.39, p.1851-1856, 2009.

PINHEIRO, L.M.S.; MARTINS, R.T.; PINHEIRO, L.A.S.; PINHEIRO, L.E.L. Rendimento industrial de filetagem da tilápia tailandesa (Oreochromis spp.). Arquivo Brasileiro de Medicina Veterinária e Zootecnia, v.58, p.257-262, 2006.

RIBAS, L.; FLOS, R.; REIG, L.; MACKENZIE, S.; BARTON, B.A.; TORT, L. Comparison of methods for anaesthetizing Senegal sole (Solea senegalensis) before slaughter: stress responses and final product quality. Aquaculture, v.269, p.250-258, 2007.
ROSS, L.G.; ROSS, B. Anaesthetic and sedative techniques for aquatic animals. 3.ed. Oxford: Blackwell Science, 2008. 240p.

ROUBACH, R.; GOMES, L.C.; FONSECA, F.A.L.; VAL, A.L. Eugenol as an efficacious anaesthetic for tambaqui, Colossoma macropomum (Cuvier). Aquaculture Research, v.36, p.1-6, 2005.

SANTOS,L.D.;ZARA, R.F.;VISENTAINER,J.V.;MATSUSHITA, M.; SOUZA, N.E.; FRANCO, M.L.R.S. Avaliação sensorial e rendimento de filés defumados de tilápia (Oreochromis niloticus Linnaeus, 1757) na presença de alecrim (Rosmarinus officinalis). Ciência e Agrotecnologia, v.31, p.406-412, 2007.

SIMÕES, L.N.; GOMES, L.C. Eficácia do mentol como anestésico para juvenis de tilápia-do-nilo (Oreochromis niloticus). Arquivo Brasileiro de Medicina Veterinária e Zootecnia, v.61, p.613-620, 2009.

SMALL, B.C. Effect of isoeugenol sedation on plasma cortisol, glucose, and lactate dynamics in channel catfish Ictalurus punctatus exposed to three stressors. Aquaculture, v.238, p.469-481, 2004.

SOUZA, M.L.R. de; MARANHÃO, T.C.F. Rendimento da carcaça, filé e subprodutos da filetagem da tilápia do Nilo, Oreochromis niloticus (L), em função do peso corporal. Acta Scientarum, v.23, p.897-901, 2001.

STOSKOPF, M. Anaesthesia. In: BROWN, L. (Ed.). Aquaculture for veterinarians: fish husbandry and medicine. London: Pergamon, 1993. p.161-168. (Veterinary handbook series).

VELÍSEK, J.; SVOBODOVÁ, Z.; PIACKOVÁ, V. Effects of clove oil anaesthesia on rainbow trout (Oncorhynchus mykiss). Acta Veterinaria Brno, v.74, p.139-146, 2005.

VIDAL, L.V.O.; ALBINATI, R.C.B.; ALBINATI, A.C.L.; LIRA, A.D. de; ALMEIDA, T.R. de; SANTOS, G.B. Eugenol como anestésico para a tilápia-do-nilo. Pesquisa Agropecuária Brasileira, v.43, p.1069-1074, 2008.

WATANABE, W.O.; LOSORDO, T.M.; FITZSIMMONS, K.; HANLEY, F. Tilapia production systems in the Americas: technological advances, trend and challenges. Reviews in Fisheries Science, v.10, p.465-498, 2002.

WOODY, C.A.; NELSON, J.; RAMSTAD, K. Clove oil as an anaesthetic for adult sockeye salmon: field trials. Journal of Fish Biology, v.60, p.340-347, 2002.

Recebido em 11 de agosto de 2010 e aprovado em 3 de novembro de 2010 